\title{
QTcB Prolongation Greater Than 500 msec
}

National Cancer Institute

\section{Source}

National Cancer Institute. QT CB Prolongation Greater Than 500 msec. NCI Thesaurus.

Code C71096.

Corrected QT interval longer than 500 msec, in accordance with Bazett's correction formula. 